\title{
Image Texture Analysis and Application to Acicular Mullite Porous Ceramic Microstructure
}

\author{
Clifford S Todd ${ }^{1}$, William A Heeschen ${ }^{1}$ \\ 1. Analytical Sciences, The Dow Chemical Company, Midland, MI, USA
}

Acicular mullite porous ceramic [1,2] can display a range of microstructures, impacted by elemental formulation, raw materials, and processing conditions. The microstructure in turn impacts performance metrics such as strength, modulus and back pressure for filtration applications. During research and development and later during scale-up and production, assessment of microstructure was done in a completely subjective manner; a small group of experienced individuals assessed SEM images. The goal of the project described here was to develop an objective way to quantify aspects of this microstructure. The approach was to apply computational image analysis to SEM images. A set of 32 samples that displayed a range of microstructures were used (Fig. 1).

There are two steps in the image analysis method developed here. First, the image is divided into grids of systematically smaller regions (domains). The mean and standard deviation of gray levels were calculated within each domain. The set of values for each domain size were combined to yield three figures of merit: The standard deviation of the average gray-levels (variability of overall image brightness); the average of the standard deviations of gray-level within the domains (overall variability of the image brightness); and the standard deviation of the standard deviations of gray-level within the domain (the variation in the brightness variability across the image). The gray-level image analysis procedure was implemented with ImageJ software using a combination of macros and plugins.

The second step in the image analysis method is to process these data using principal component analysis, implemented here using MatLab ${ }^{\circledR}$ software. The data were a matrix of 64 x 24 (32 samples, two image areas per sample $x$ three figures of merit at eight domain sizes each). The model had four significant Principal Components based on the size of the eigenvalues. Principal Component 1 (PC1) accounted for nearly $81 \%$ of the variability in the original data. PC2 accounted for over $10 \%$ of the variability.

From a manufacturing standpoint, it is important that microstructure is consistent within a material so that consistent material properties result. Therefore a narrow size distribution of mullite crystals and/or pore size is preferred as opposed to a bimodal distribution. Well-formed individual mullite crystals are preferred, as opposed to rough agglomerates of mullite crystal. A large amount of a non-acicular (nonmullite) phase is not desired because the backpressure and filtration advantages of this ceramic are a direct result of the acicular crystal structure; any non-acicular phase reduces the impact of this differentiation. Images of the 32 samples used for this study were subjectively rated by trained individuals on a scale from 1 (inconsistent microstructure) to 5 (very consistent microstructure). A rating of 3 was considered minimally consistent.

Figure 2 shows that PC2 scores are inversely correlated to microstructure consistency rating. Statistical analysis indicated that PC1 and PC3 scores were not significant in the correlation, and PC4 scores did not improve the fit much. A simple criterion is that microstructure is acceptably consistent if the score of PC2 is less than zero. 
Figure 3 shows that for samples with acceptably consistent microstructure, PC1 scores are positively correlated to average mullite grain size, as measured manually from the SEM images. Statistical analysis indicated that PC2 and PC4 scores were not significant in the correlation, and PC3 scores did not improve the fit much.

The developed image texture analysis method was successful in objectively quantifying the microstructure of acicular mullite porous ceramic. Texture analysis metrics correlated with the important parameters of mullite crystal size (and presumably pore size) and microstructure consistency.

References:

[1] Pyzik et al, J European Ceramic Society 28 (2008) p.383.

[2] Pyzik et al, PACRIM 11 conference (2015).

\begin{tabular}{|c|c|c|c|}
\hline $\begin{array}{l}\text { Figure 1. Example } \\
\text { SEM images of cera- } \\
\text { mic. Field of view is } \\
345 \mu \mathrm{m} \text {. (A) Large } \\
\text { grain size, consistent } \\
\text { microstructure. (B) } \\
\text { Small size, consistent. } \\
\text { (C) Inconsistent } \\
\text { microstructure. }\end{array}$ & 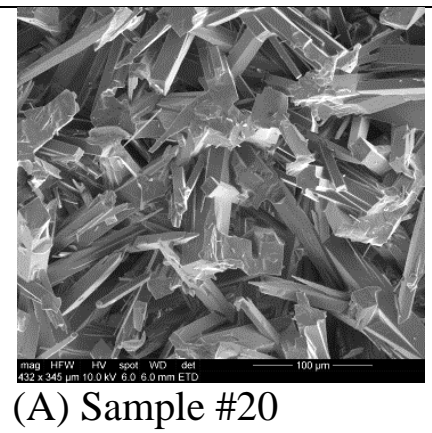 & $\begin{array}{l}\text { (u) } \\
\text { (B) Sample \#29 }\end{array}$ & 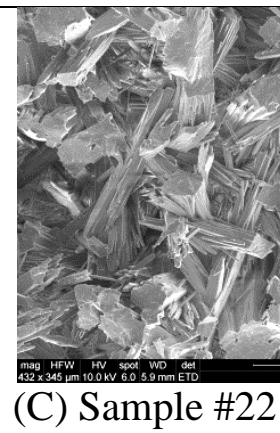 \\
\hline
\end{tabular}

\begin{tabular}{|c|c|c|c|c|c|c|c|c|c|}
\hline $\begin{array}{l}\text { Figure 2. (A) PC1 } \\
\text { scores versus PC2 } \\
\text { scores, coded by } \\
\text { subjective micro- } \\
\text { structure consistency } \\
\text { (MC). (B) Plot of } \\
\text { microstructure } \\
\text { consistency as a } \\
\text { function of PC2 score. }\end{array}$ & 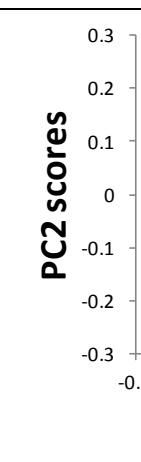 & 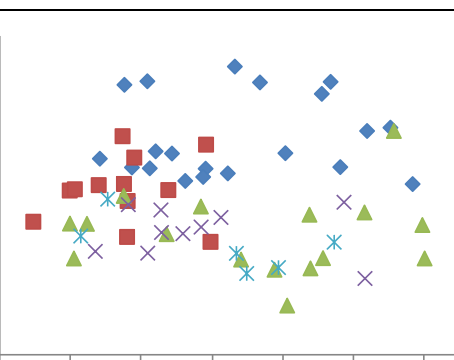 & $\begin{array}{l}\text { (A) } \\
-\mathrm{MC1} \\
\square \mathrm{MC2} \\
\triangle \mathrm{MC3} \\
\times \mathrm{MC4} \\
* \mathrm{MC5}\end{array}$ & $\begin{array}{r}5 \\
4 \\
\Sigma 3 \\
2 \\
1\end{array}$ & & \multicolumn{3}{|c|}{ 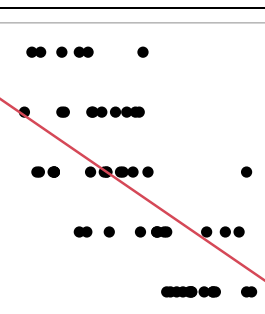 } & \\
\hline
\end{tabular}

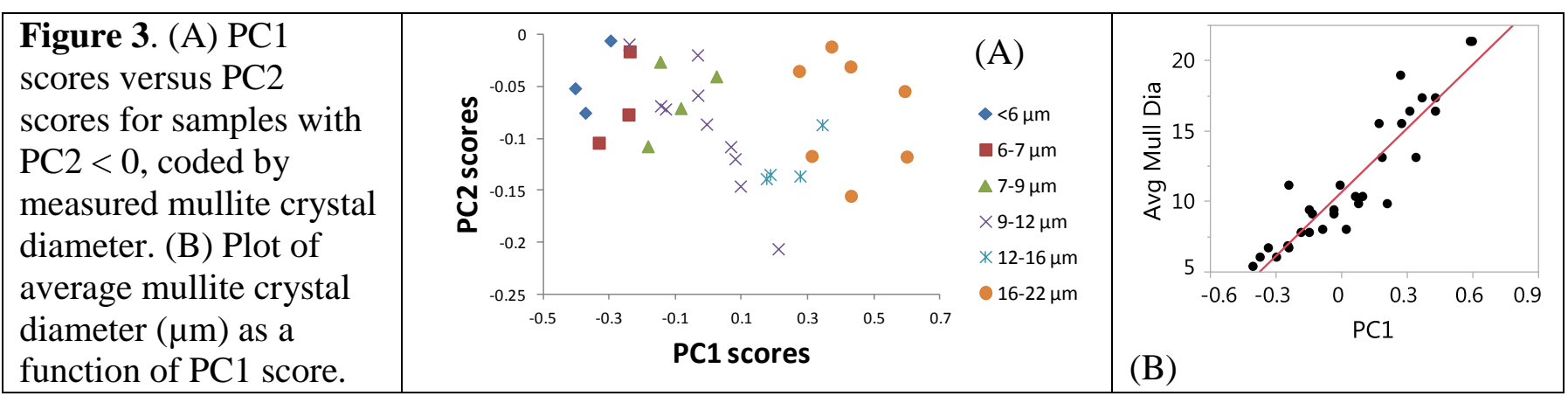

